
\title{
25 Research Soure \\ Sodium-fluorescein Guided Resection in Brain Metastases Surgery: Our Results in a Series of 59 Patients
}

Dalila Fuschillo ( $\nabla$ dalilafuschillo@gmail.com )

Maurizio Bufalini Hospital: Ospedale Maurizio Bufalini https://orcid.org/0000-0003-4622-925X

Lorenzo Mongardi

University of Ferrara: Universita degli Studi di Ferrara

Marcello D'Andrea

Maurizio Bufalini Hospital: Ospedale Maurizio Bufalini

Giuseppe Maimone

Maurizio Bufalini Hospital: Ospedale Maurizio Bufalini

Vincenzo Antonelli

Maurizio Bufalini Hospital: Ospedale Maurizio Bufalini

Luigino Tosatto

Maurizio Bufalini Hospital: Ospedale Maurizio Bufalini

\section{Research Article}

Keywords: Metastasis, tumors in the brain, sodium fluorescein, metastatic tumor surgery

Posted Date: June 18th, 2021

DOI: https://doi.org/10.21203/rs.3.rs-599819/v1

License: () (1) This work is licensed under a Creative Commons Attribution 4.0 International License.

Read Full License 


\section{Abstract}

Metastasis are the most common tumors in the brain, nowadays surgical resection still remains the treatment of choice in patients eligible for surgery.

Several tools and techniques have been proposed to improve the resection rate but few datas are available on the application of fluorescence guided surgery for brain metastases resection

Purpose: the aim of the study was to assess the safety and efficacy of the fluorescence-guided surgery through the application of sodium fluorescein (SF) detected by Yellow $560 \mathrm{~nm}$ filter for brain metastases surgery

Methods: 75 Patients operated from January 2015 to January 2020 were retrospectively analyzed and 59 patients recruited. The patients were divided into group A of 30 (fluorescein-guided surgery group of 30 patients) and group B (control- standard white light microsurgery of 29 patients). In group A a $5 \mathrm{mg} / \mathrm{kg}$ bodyweight of FL was intravenously injected at scalp incision. A yellow 560 filter was employed for microsurgical tumor resection. Preoperative and postoperative metastases volume analysis was performed to evaluate the extent of resection and the residual tumor rate

Results: Median preoperative Volume (cm3) was 11.85 (range 2.5-90) in group A and 10 (range 1-48) in group B. Median residual tumor volume (RTV) after surgery was 0 (0-1.0) in group A and 0.4 (0-2.0) in group B whereas median EOR was $100 \%(0,8-1)$ in the group A and $98,2 \%(0,43-1)$ in group $B$.

Conclusion: SF-guided surgery using YELLOW $560 \mathrm{~nm}$ microscope filter should be considered a safe and useful procedure and effective to improve the extent of resection even in metastatic tumor surgery.

\section{Introduction}

Metastasis are the most common tumor affecting the brain: lung cancer, breast cancer and melanoma, in particular, are the most common solid tumors spreading to the central nervous system (CNS).

In previous published studies it has been estimated that $9-17 \%$ of cancer patients will develop brain metastases during the course of their disease and that approximately half will die within 3-27 months from the initial diagnosis of CNS involvement [1-3].

Individualized therapies and new understandings of the molecular mechanisms of brain metastasis are leading to a progressive increase of the survival but tumor removal still plays a fundamental role in patients eligible for surgery.

In the last decades several tools and techniques have been proposed to improve the extent of resection in surgery for malignant tumors as the fluorescent dye 5-aminolevulinic acid (5-ALA), first described by Stummer et al in 2006 and then by other Authors for high grade gliomas [4-6]; however its application in brain metastasis surgery had been proved to be ineffective [7-11]. 
SF is a salt and an organic fluorescent dye with peak excitation at $490 \mathrm{~nm}$ and emission between 500 and 550nm widely used in several fields of medicine: after endovenous administration, SF accumulates in the tumor tissue through the dysfunctional blood-brain barrier (BBB) demarcating the lesion [12].

The use of this dye is known in neurosurgery since 1947 when G.E.Moore et al reported their first experience in a series of 46 patients [13].

Some authors reported their impressions in the application of fluorescein sodium for the resection of brain metastasis with promising results [14-24].

Herein we report our experience in fluorescence-guided surgery through the application of sodium fluorescein detected by Yellow 560nm (PENTERO 900, Carl Zeiss, Meditec, Oberkochen, Germany) in a brain metastasis surgical series of 30 patients. These cases have been compared with a control group of 29 patients operated with bright-light surgery and our results integrated with current literature.

\section{Methods}

We report a retrospective study involving 59 patients affected with brain metastasis (BMs) surgically treated at Neurosurgery Department at"M.Bufalini” Hospital, Cesena Italy.

Patients operated on from January 2015 to January 2020 were retrospectively recruited.

Inclusion criteria were: age 18 years and over, singular or multiple CMs and life expectancy of more than 3 months, Karnofsky performance scale (KPS) 60 or more and adequate pre and post operative neuroimaging.

All patients underwent pre and postoperative (within the first 48hours) CT scan and/or MRI (1,5T Siemens) including 3D volumetric sequences with and without contrast.

Demographic and clinical characteristics were recorded for all patients, including age, gender, histology ; post-operative tumor resection rate EOR and fluorescein related complications (Tab 1 and 2).

Informed consent was obtained from patients for the off-label use of FL.

FL (5 mg/kg body weight) was injected intravenously in group $A$ at scalp incision; after the administration , the tumor staining was visualized with the use of a specific microscope filter (YELLOW 560-nm filter, Pentero 900, ZEISS Meditec, Germany).

Surgical resection was performed using both white light and yellow-560 nm filter for the identification of fluorescence enhancing tumor and to evaluate the border with normal brain parenchima (Fig.1).

In group $B$, the standard white light microsurgical procedure was carried out for resection of tumors. 
In both groups, when required, adjunctive tools such as neuronavigation and intraoperative monitoring were adopted.

Surgeon's opinion and satisfaction after surgery (expressed as useful or not useful), were recorded; the extent of resection was evaluated by calculating the pre-operative tumor volume (PTV) on MRI scan and the residual tumor volume (RTV) in the immediate post-operative.

The volume segmentation was performed by using a quantitative, semiautomated software tool (Myrian, Intrasense, fig 2), and the analysis was performed independently by two authors (GM, LM) blinded to each other's results.

The MRI images were obtained by a 1.5 T Philips scanner, including 3D pre- and post-enhancement T1 scans and T2 FLAIR.

The extent of resection (EOR) was calculated by using the following formula: (PTV - RTV)/PTV. All volume values were expressed in $\mathrm{cm} 3$.

We performed statistical analysis (IBM SPSS statistics 25) comparing PTV lesions and the RTV in the two groups with Pearson correlation coefficient, and $p$ values were reported for each variable. A Fisher exact test was adopted to correlate the EOR with the histology of the metastases by dicothomically grouping FLG and BL based on the presence of a post-operative residual tumor (cut-off: residual volume less than $0.5 \mathrm{~cm} 3)$.

We also performed an overall survival analysis by calculating the period from the initial surgery to the time of death of the patients by using the Kaplan-Meier method and the significance in difference among survival curves (Fig 3).

\section{Results}

We collected data of 30 patients (9 M $21 \mathrm{~F}$ ) who underwent MS resection via Fluorescence-guided surgery (FGS); this group was compared with a cohort of 29 MS patients (13M $16 \mathrm{~F}$ ) who underwent the traditional white light surgery $(\mathrm{BL})$.

The median age at surgery was 63.5 years in the FGS group and 61.0 years in BL group.

MSs were located in the frontal lobe in 20 cases, in the temporal lobe in 11 cases, in the parietal lobe in 11 cases, in the occipital lobe in 5 cases, in the cerebellum in 12 cases.

The most common MSs origins were: lung metastasis (8 patients in FGS group vs. 7 patients in BL group), breast metastasis (9 patients in FGS group vs. 5 patients in BL group), and melanoma metastasis (2 patients vs in FGS group vs 6 patients in BL group). The groups are shown in Table 1.

\section{Volume Measurements and Fluorescence Characteristics}


Median PTV was $11.85 \mathrm{~cm} 3$ (range $2.5-90 \mathrm{~cm} 3$ ) in the FG group and $10 \mathrm{~cm} 3(1-48 \mathrm{~cm} 3)$ in the BL group. Median RTV after surgery was $0(0-1.5 \mathrm{~cm} 3)$ in the $F G$ group and $0.4 \mathrm{~cm} 3(0-4.0 \mathrm{~cm} 3)$ in the $\mathrm{BL}$ group, whereas median EOR was 100\% (0.8-1) in the FG group and $98.2 \%(0,43-1)$ in the BL group. All data are reported in Table 2.

The vast majority of tumors $(27 / 30,90 \%)$ showed an intense fluorescent yellow-green signal, whereas the remnant cases $(3 / 30,10 \%)$ showed insufficient FL staining (1 melanoma, 1 lung, and 1 colorectal Ms). Fluorescein-related complications were not reported.

Surgeons evaluated the fluorescein application in MSs resections as useful in almost all cases.

\section{Volume Correlations and Survival Curves}

We perform Pearson correlation analysis to evaluate the degree to which the above-mentioned variables are interdependent. For instance, EOR is clearly inversely related to RTV, but both also depend on the PTV. The only strong statistical correlations we found were between RTV and EOR in both FLG $(r=-0.81)$ and $B L(r=-0.75)$ groups $(p<0.0001)$. The remaining volumes were not statistically significant. The survival analysis through Kaplan-Meyer curve was calculated in 15 months and did not show significant difference (Fig. 1). The Fisher test did not show significant results in the three group metastases in which we performed the analysis (Lung $p=0.16$, Breast $p=0.37$, and Melanoma $p=0.38$ ).

\section{Systematic Review Of The Literature}

Systematic review of the Literature

A review of the current Literature, according with the PRISMA guidelines, regarding the application of fluorescein for the resection of brain metastasis was performed [25].

Using Pubmed MeSH database, Cochrane and Embase all English papers published until May 2020, including words "metastasis", "fluorescein sodium", "resection”, "fluorescence guided resection” "YELLOW 560 NM FILTER" were reviewed. Each article was carefully scrutinized in order to select those in which a detailed description of the Fluorescein sodium dosage/use and intraoperative fluorescein related findings were reported. A double cross-reference check of papers considered for eligibility (forward search) was performed, in order to include supplementary papers erroneously undetected in the first search round.

Inclusion criteria: English written papers including human participants (age > 18 years); availability of clinical and demographic data of the included patients; a clear description of fluorescence dosage/use and intraoperative fluorescein related findings.

Exclusion Criteria: incomplete clinical data; lack of fluorescence dosage/use and intraoperative fluorescein related findings; editorials and commentaries; papers with less the 5 cases where excluded.

From the first literature research, we retrieved 21 articles. After duplicates removal and titles/abstract screening, 16 papers were considered for eligibility. Six papers were then excluded with the following 
reasons: case reports and opinion articles. Finally, 10 studies (290 patients) matched the inclusion criteria, and were included in the present review [15-24].

The literature review comprises 290 patients diagnosed of brain metastasis and surgically treated through the application of fluorescein sodium.

In just one article [15] the authors reported a preoperative evaluation of MSs dimensions through the diameter.; the extent of resection was defined as GTR (no residual tissue) or STR (with residual tumor tissue). The first was achieved in $192 / 222$ reported cases (86.4\%), while STR in 30 cases $(15.6 \%)$.

No fluorescein related complications were reported.

Six authors reported a subjective satisfaction rate of $100 \%$ in the fluorescein use for the resection on MSs, while 3 authors (all with a satisfaction rate $>90 \%$ ) reported a insufficient fluorescein staining in 9 cases (5 lung, 2 melanomas , 1 renal and 1 unspecified); 1 Author didn't report this data.

Fluorescein dosage and time of injection are quite different in almost every study.

All of the Authors concluded that fluorescein-guided surgery is a safe method that can lead to higher proportion of resection compared to common microsurgery.

\section{Discussion}

Metastasis are the most common brain tumor and nearly $20 \%$ of patients with cancer will develop brain metastases through the spreading of tumoral cells in cerebral microcirculation.

Although sistemic therapies are limited in efficacy, these tumors must be treated in a multimodal way and surgical removal is indicated in patients with singular lesion.

Even if metastases are not primary cerebral tumors, the boundary between the lesion and normal brain parenchima is not always clear even though the use of some tools as neuronavigation and intraoperative ultrasound have improved the post-operative results.

Complete surgical resection is advocated; in fact, some Authors demonstrated the correlation between the extent of resection and recurrence rate, progression-free survival, overall survival and quality of life $[7,24$, $26,27]$.

The use of fluorescein sodium salt was successfully applied in glioma surgery [28];fluorescein accumulates in pathological tissues through the damaged blood-brain barrier enhancing the lesion and making it easier to remove.

The same technique can be applied in metastases surgery; some Authors $[15,17,18]$ showed that this tool leads to higher proportion of resection compared to white light surgery, minimizing normal tissue 
manipulation; nevertheless, none of these studies reported a volumetric analisys and the extent of resection was indicated as gross total or subtotal removal (absence or presence of residual tumor).

Our data confirmed that the i.v use of fluorescein in metastases surgery is safe with no adverse effect in our group of patients.

We evaluated the influence of FGS on resection extension in two groups of patients with CMs and we found that residual tumor tissue median is zero in FGS group with $100 \%$ of median EOR comparing with the $98.2 \%$ in BL-surgery group. This volumetric datas strongly support the usefulness of this method in increasing the extent of resection as already mentioned by some Authors.

We reported a good fluorescein staining in almost $90 \%$ of patients as reported also by Schebesh et al. [18, 19] ; further on, in our analysis we performed Fisher exact test to determine variations in tumor staining in different hystologies. We did not found significant statistical correlations, but in accord to surgeons' impression, we found a possible benefit by using fluorescence with lung metastases $(p=0.16)$. This data, which should be carefully confirmed in further statistical studies, could identify some histological subtypes that could take advantage more than others from this dye.

Moreover, previous studies reported a great heterogeneity regarding the time of fluorescein injection and the dose to be administered; at this time it's not possible to determine objectively which is the best protocol to applicate to obtain the best results in term of major definition of normal brain parenchima/tumor interface and in term of adverse effect.

Preliminary data on overall survival are evaluated by assessing KM method: till now we didn't find any statistically difference among survival curves. However, due to the short follow up time in our cohort we can't draw any conclusion about long-term outcome. The limited number of patients and other parameters that affected survival may explain the lack of statistical significance for median survival time.

\section{Conclusions}

As far as we know this is the first study to report objective datas on the extent of resection of metastatic tumors through the calculation of the residual tumor volume in two CM surgery groups (FGS vs $B L$ ).

As being concluded in previous publications this study confirmed that SF- guided surgery with the use of YELLOW $560 \mathrm{~nm}$ microscope filter is safe, effective and useful to improve extent of resection even in metastatic tumor surgery.

However, to confirm the efficacy and to prove the impact on patient survival and quality of life and the better specificity of this dye on some hystological type of tumors, prospective randomized studies with larger patient samples and with long-term follow-up need to be done. 


\section{Declarations}

COMPLIANCE WITH ETHICAL STANDARDS:

FUNDING

The authors did not receive support from any organization for the submitted work.

No funding was received to assist with the preparation of this manuscript.

No funding was received for conducting this study.

No funds, grants, or other support was received.

CONFLICTS OF INTEREST

The authors have no relevant financial or non-financial interests to disclose.

The authors have no conflicts of interest to declare that are relevant to the content of this article.

All authors certify that they have no affiliations with or involvement in any organization or entity with any financial interest or non-financial interest in the subject matter or materials discussed in this manuscript.

The authors have no financial or proprietary interests in any material discussed in this article.

ETHICS APPROVAL

All procedures performed in studies involving human participants were in accordance with the ethical standards of the institutional research committee and with the 1964 Helsinki declaration and its later amendments or comparable ethical standards.

INFORMED CONSENT

Informed consent was obtained from all individual participantsincluded in the study

DATA AVAILABILITY STATEMENT:

AVAILABILITY OF DATA AND MATERIAL

Data available on request from the Authors

CODE AVAILABILITY

Not applicable

AUTHOR CONTRIBUTION: 
- Dalila Fuschillo MD: Primary author and corresponding author. Email address: dalilafuschillo@gmail.com ORCID: 0000-0003-4622-925X

Lorenzo Mongardi MD: Data collection and reviewer of the literature ORCID 0000-0002-1374-5736

Marcello D'Andrea MD: Revisor of the scientific content of the manuscript ORCID: 0000-0003-0894-7509

Giuseppe Maimone MD: Data analysis and interpretation

Vincenzo Antonelli MD: Grammatical revisions to manuscript ORCID: 0000-0003-4231-529X

Luigino Tosatto MD: Head of project

\section{References}

1 Nayak L, Lee EQ, Wen PY (2012) Epidemiology of brain metastases. Curr Oncol Rep 14:48-54. https://doi.org/10.1007/s11912-011-0203-y

2 Brastianos PK, Curry WT, Oh KS (2013) Clinical discussion and review of the management of brain metastases. J Natl Compr Canc Netw 11:1153-64. https://doi.org/10.6004/jnccn.2013.0133

3 Ferguson SD, Zheng S, Xiu J, Zhou S, Khasraw M et al (2018) Profiles of brain metastases: Prioritization of therapeutic targets. Int J Cancer 143: 3019-3026. https://doi.org/10.1002/ijc.31624

4 Stummer W, Pichlmeier U, Meinel T, Wiestler OD, Zanella F, Reulen HJ, Group AL-GS (2006) Fluorescence-guided surgery with 5 -aminolevulinic acid for resection of malignant glioma: a randomised controlled multicentre phase III trial. Lancet Oncol 7: 392-401. https://doi.org/10.1016/S14702045(06)70665-9

5 Kuroiwa T, Kajimoto Y, Ohta T (1998) Development of a fluorescein operative microscope for use during malignant glioma surgery: a technical note and preliminary report. Surg Neurol 50: 41-49. https://doi.org/10.1016/s0090-3019(98)00055-x

6 Kuroiwa T, Kajimoto Y, Ohta T (1999) Comparison between operative findings on malignant glioma by a fluorescein surgical microscopy and histological findings. Neurol Res 21:130-134. https://doi.org/10.1080/01616412.1999.11740909

7 Gates M, Alsaidi M, Kalkanis S (2012) Surgical treatment of solitary brain metastases. Prog Neurol Surg 25:74-81. https://doi.org/10.1159/000331178

8 Lee CH, Kim DG, Kim JW, Han JH, Kim YH et al (2013) The role of surgical resection in the management of brain metastasis: a 17-year longitudinal study. Acta Neurochir 155:389-397. https://doi.org/10.1007/s00701-013-1619-y 
9 Patchell RA, Tibbs PA, Walsh JW, Dempsey RJ, Maruyama Y et al (1990) A randomized trial of surgery in the treatment of single metastases to the brain. N Engl J Med 322:494-500.

https://doi.org/10.1056/NEJM199002223220802

10 Kamp MA, Grosser P, Felsberg J, Slotty PJ, Steiger HJ, Reifenberger G, Sabel M (2012) 5-aminolevulinic acid D5-ALA]- induced fluorescence in intracerebral metastases: a retrospective study. Acta Neurochir (Wien) 54:223-228. https://doi.org/10.1007/s00701-011-1200-5

11 Marbacher S, Klinger E, Schwyzer L, Fischer I, Nevzati E, Diepers M, Roelcke U, Fathi AR, Coluccia D, Fandino $\mathrm{J}$ (2014) Use of fluorescence to guide resection or biopsy of primary brain tumors and brain metastases. Neurosurg Focus 36:E10. https://doi.org/10.3171/2013.12.FOCUS13464

12 R. Sjöback, J. Nygren, M. Kubista (1995) Absorption and fluorescence properties of fluorescein. Spectrochem. Acta A Mol Biomol Spectrosc. 51: L7-L21. https://doi.org/10.1016/0584-8539(95)01421-P

13 Moore GE (1947) Fluorescein as an agent in the differentiation of normal and malignant tissues. Science 106:130-131. https://doi.org/10.1126/science.106.2745.130-a

14 Moore GE, Peyton WT, French LA, Walker WW (1948) The clinical use of fluorescein in neurosurgery: the localization of brain tumors. J Neurosurg 5:392-398. https://doi.org/10.3171/jns.1948.5.4.0392

15 Okuda T, Kataoka K, Yabuuchi T, Yugami H, Kato A (2010) Fluorescence-guided surgery of metastatic brain tumors using fluorescein sodium. Journal of clinical neuroscience 17:118-121. https://doi.org/10.1016/j.jocn.2009.06.033

16 Okuda T, Kataoka K, Taneda M (2007) Metastatic brain tumor surgery using fluorescein sodium: technical note. Minim Invasive Neurosurg 50:382-4. https://doi.org/10.1055/s-2007-993200

17 Höhne J, Hohenberger C, Proescholdt M, Riemenschneider MJ, Wendl C, Brawanski A, Schebesch KM (2017) Fluorescein sodium- guided resection of cerebral metastases-an update. Acta Neurochir 159:363367. https://doi.org/10.1007/s00701-016-3054-3

18 Schebesch KM, Hoehne J, Hohenberger C, Proescholdt M, Riemenschneider MJ, Wendl C, Brawanski A (2015) Fluorescein sodium- guided resection of cerebral metastases-experience with the first 30 patients. Acta Neurochir 157:899-904. https://doi.org/10.1007/s00701-015-2395-7

19 Schebesch KM , Proescholdt M, Hohne J, Hohenberger C, Hansen E, Riemenschneider MJ, Ullrich W, Doenitz C et al (2013) Sodium fluorescein-guided resection under the YELLOW $560 \mathrm{~nm}$ surgical microscope filter in malignant brain tumor surgery--a feasibility study. Acta Neurochir (Wien) 155:693-9. https://doi.org/10.1007/s00701-013-1643-y

20 Xiao SY, Zhang J, Zhu ZQ, Li YP, Zhong WY, Chen JB, Pan ZY, Xia HC (2018) Application of fluorescein sodium in breast cancer brain-metastasis surgery. Cancer management and research 10: 4325-4331. 
21 Hamancioglu MK, Akçakaya MO, Goker B, Kasimcan MO, Kiris T (2016) The use of the YELLOW 560 $\mathrm{nm}$ surgical microscope filter for sodium fluorescein-guided resection of brain tumors: Our preliminary results in a series of 28 patients. Clinical Neurology and Neurosurgery 143: 39-45.

https://doi.org/10.1016/j.clineuro.2016.02.006

22 Gollapudi PR, Mohammed I, Pittala SR, Kotha AR, Reddycherla NR, Ginjupally DR (2018) A simple improvisation to enhance the utility of Fluorescein sodium in the resection of intracranial lesions at routine neurosurgical centres. World Neurosurgery 112: 14-17.

https://doi.org/10.1016/j.wneu.2018.01.039

23 Falco J, Cavallo C, Vetrano I, De Laurentis C, Siozos L, Schiariti M, Broggi M, Ferroli P, Acerbi F (2019) Fluorescein application in cranial and spinal tumors enhancing at preoperative MRI and operated with a dedicated filter on the surgical microscope: preliminary results in 279 patients enrolled in the FLUOCERTUM prospective study. Frontiers in surgery 6:49.

https://doi.org/10.3389/fsurg.2019.00049.eCollection

24 Kofoed MS, Pedersen CB, Schulz MK, Kristensen BW, Hansen RW, Markovic L, Halle B, Poulsen FR (2021) Fluorescein-guided resection of cerebral metastases is associated with greater tumor resection. Acta Neurochir Epub ahead of print. https://doi.org/10.1007/s00701-021-04796-1

25 Moher D, Liberati A, Tetzlaff J, Altman DG, PRISMA Group (2009) Preferred reporting items for systematic reviews and meta-analyses: the PRISMA statement. PLos Medicine 6:e1000097. https://doi.org/10.1371/journal.pmed.1000097

26 Lee CH, Kim DG, Kim JW, Han JH, Kim YH, Park CK, Kim CY, Paek SH, Jung HW (2013) The role of surgical resection in the management of brain metastasis: a 17-year longitudinal study. Acta Neurochir (Wien) 155:389-397. https://doi.org/10.1007/s00701-013-1619-y

27 Kamp MA, Dibue M, Niemann L, Reichelt DC, Felsberg J, Steiger HJ, Szelenyi A, Rapp M, Sabel M (2012) Proof of principle: supramarginal resection of cerebral metastases in eloquent brain areas. Acta Neurochir (Wien) 154:1981-1986. https://doi.org/10.1007/s00701-012-1463-5

28 Acerbi F, Broggi M, Eoli M, Anghileri E, Cavallo C, Boffano C, Cordella R, Cuppini L, Pollo B, Schiariti M, Visintini S, Orsi C, La Corte E, Broggi G, Ferroli P (2014) Is fluorescein-guided technique able to help in resection of high-grade gliomas? Neurosurg Focus 36:E5. http://doi.org/10.3171.2013.11.FOCUS13487

\section{Tables}

\section{TABLE 1 DEMOGRAPHIC TABLE}




\begin{tabular}{|c|c|c|c|}
\hline & & GROUP A & GROUP B \\
\hline N. OF PATIENTS & & 30 & 29 \\
\hline MEDIAN AGE & & 63.5 & 61.0 \\
\hline$M / F$ & & $9 / 21$ & $13 / 16$ \\
\hline \multicolumn{4}{|l|}{ LOCALIZATION } \\
\hline & FRONTAL & 10 & 10 \\
\hline & TEMPORAL & 5 & 6 \\
\hline & PARIETAL & 5 & 6 \\
\hline & OCCIPITAL & 3 & 2 \\
\hline & CEREBELLAR & 8 & 1 \\
\hline \multicolumn{4}{|l|}{ ORIGIN } \\
\hline & BREAST & 9 & 5 \\
\hline & LUNG & 8 & 7 \\
\hline & MELANOMA & 4 & 7 \\
\hline & OVARIAN & 2 & 1 \\
\hline & COLON & 3 & 3 \\
\hline & OTHERS & 4 & 5 \\
\hline COMPLICATION FLUO RATE & & 0 & I \\
\hline
\end{tabular}

TABLE 2 STATISTICAL DATAS

\begin{tabular}{|lll|}
\hline & GROUP A & GROUP B \\
\hline NUMBER OF PATIENTS & 30 & 29 \\
\hline Median PTV (cm3) & 11.85 & 10 \\
\hline Range & $2.5-90$ & $1-48$ \\
\hline Median RTV & 0 & 0.4 \\
\hline Range & $0-1.5$ & $0-4.0$ \\
\hline Median EOR & $100 \%$ & $98.2 \%$ \\
\hline Range & $0.8-1$ & $0.4286-1$ \\
\hline
\end{tabular}




\section{Figures}

\section{INTRAOPERATIVE MICROSCOPICAL IMAGING}

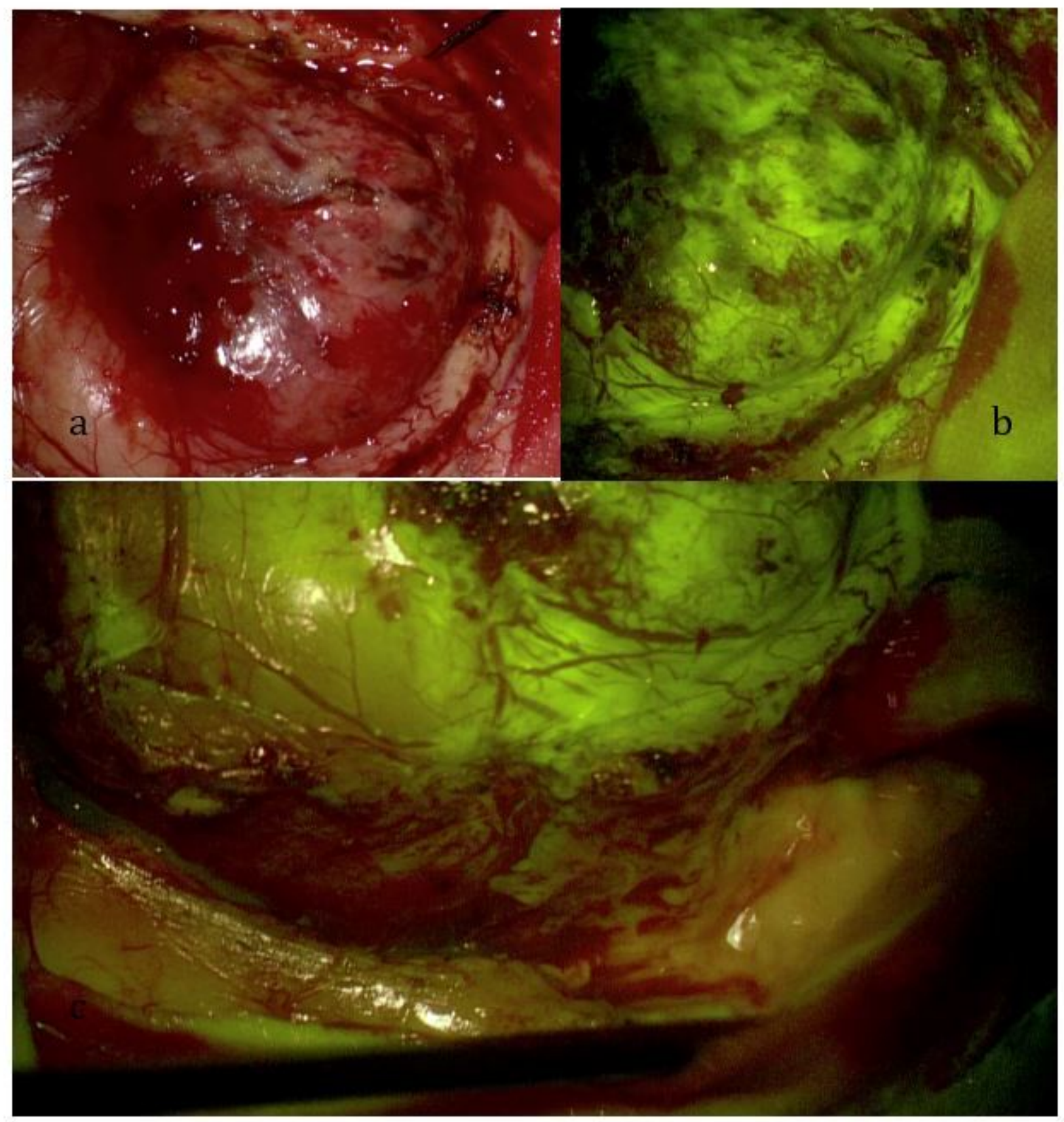

Figure 1

a) before Yellow 560 filter b) after Yellow 560 filter c) showing the difference staining between tumor and normal brain parenchima 


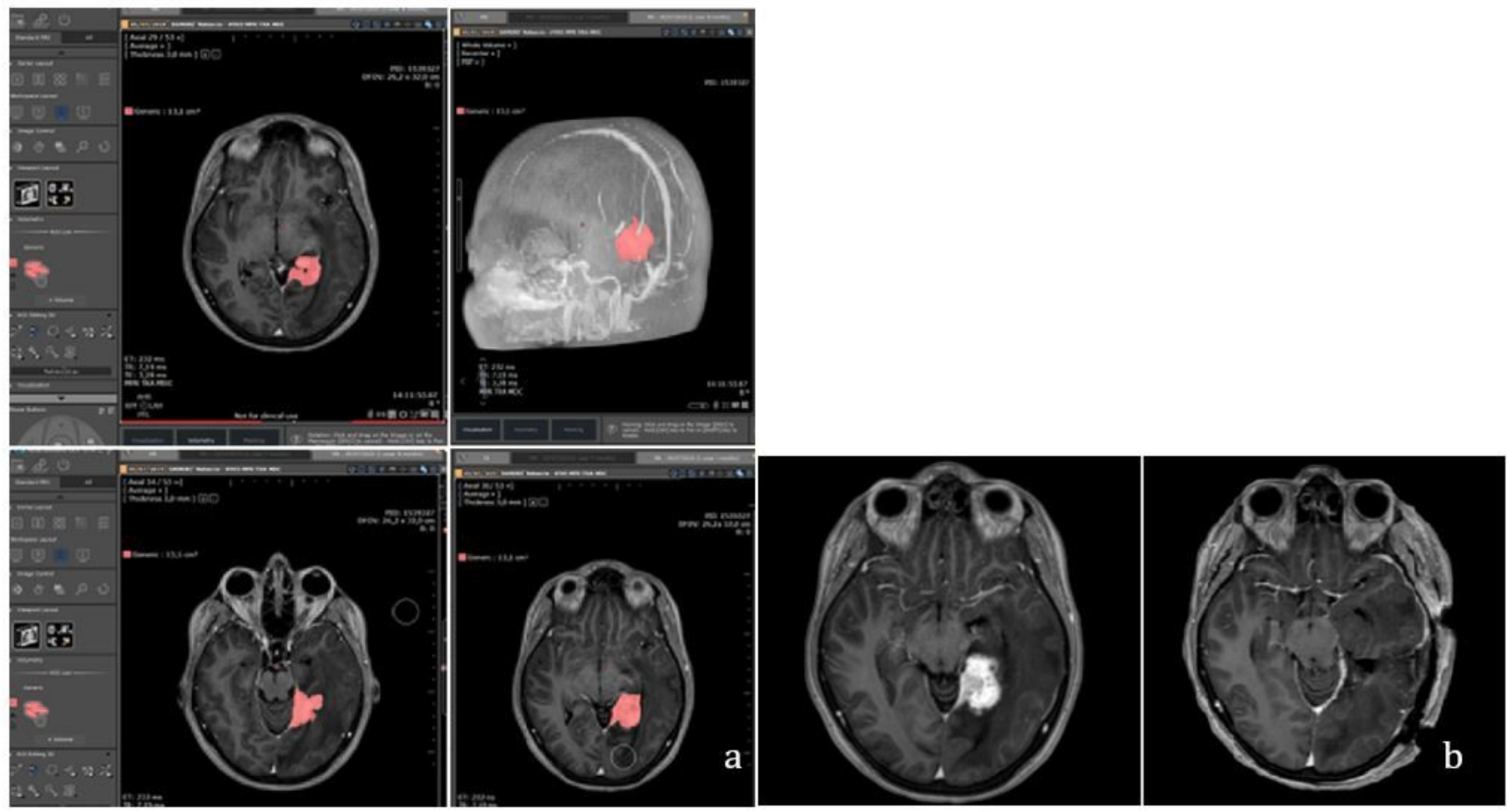

Figure 2

a) Volume segmentation performed by using a quantitative, semiautomated software tool (Myrian, Intrasense) b) Pre and Post-operative MRI 


\section{SURVIVAL ANALYSIS}

\section{Survival Curve}

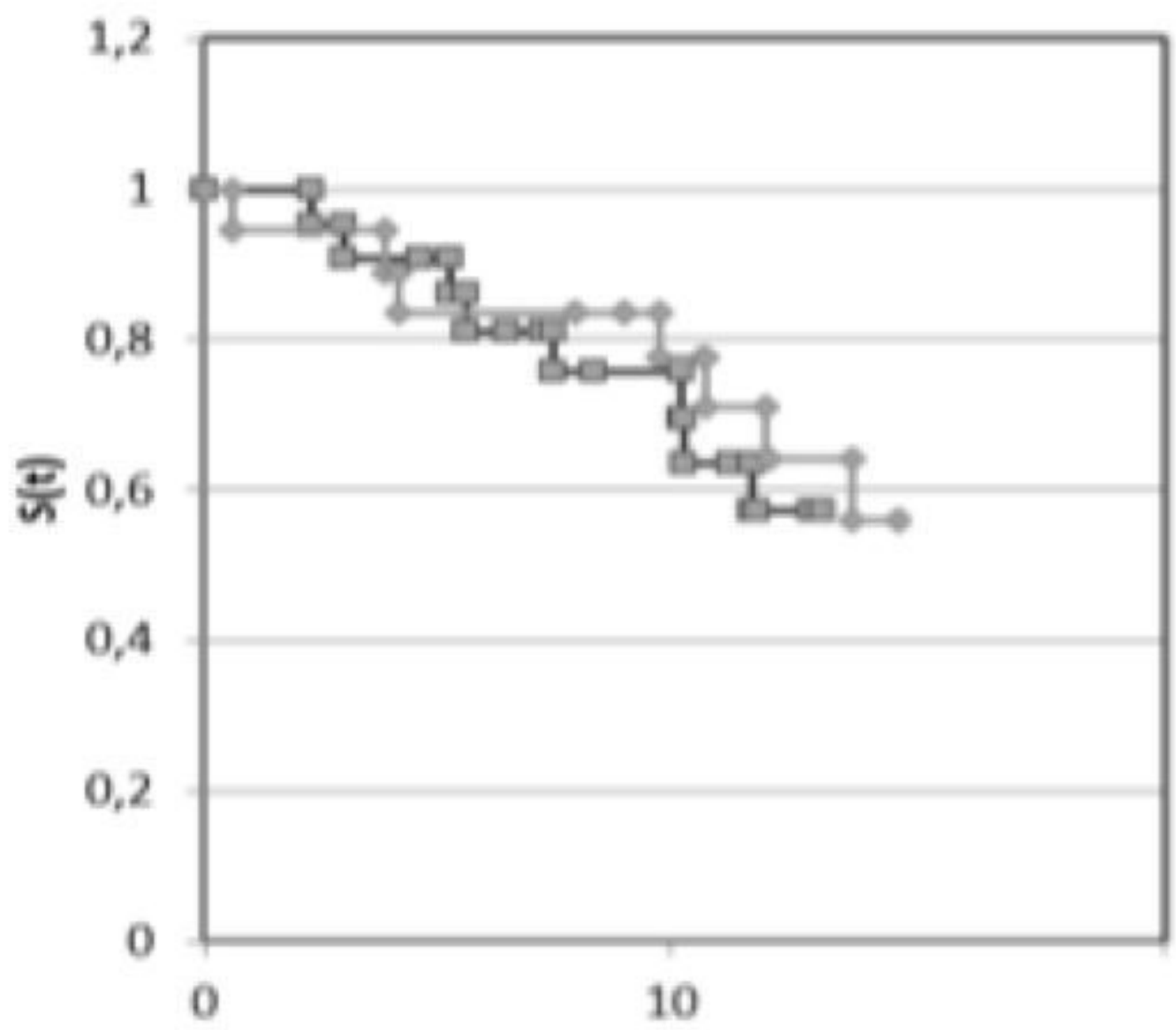

Time t

\section{Figure 3}

We also performed an overall survival analysis by calculating the period from the initial surgery to the time of death of the patients by using the Kaplan-Meier method and the significance in difference among survival curves (Fig 3). 